\title{
Using Degraded Music Quality to Encourage a Health Improving Walking Pace: BeatClearWalker
}

\author{
Andreas Komninos ${ }^{1}$, Mark D Dunlop ${ }^{1}$, David Rowe ${ }^{2}$, Allan Hewitt ${ }^{2}$, Steven Coull ${ }^{1}$ \\ Computer \& Information Sciences \\ University of Strathclyde \\ Glasgow UK \\ \{andreas.komninos,mark.dunlop\}@strath.ac.uk \\ Physical Activity for Health Research Group \\ University of Strathclyde \\ Glasgow UK \\ \{david.rowe, allan.hewitt\}@ strath.ac.uk
}

\begin{abstract}
Meeting the target of 8000 steps/day, as recommended by many national governments and health authorities, can provide considerable physical and mental health benefits and is seen as a key target for reducing obesity levels and improving public health. However, to optimize the health benefits, walking should be performed at a "moderate" intensity. While there are numerous mobile fitness applications that monitor distance walked, none directly support walking at this cadence nor has there been any research into live feedback for walking cadence. We present a smartphone fitness application to help users learn how to walk at a moderate cadence and maintain that cadence. We apply real-time audio effects that diminish the audio quality of music when the target walking cadence is not being reached. This provides an immersive and intuitive application that can easily be integrated into everyday life as allows users to walk while listening to their own music and encourages eyes-free interaction. In this paper, we introduce our approach, design, initial lab evaluation and a controlled outdoor study. Results show that using music degradation decreases the number of below-cadence steps, that users felt they worked harder with our player and would use it while exercise walking.
\end{abstract}

Keywords - Exercise; physical activity; healthy lifestyle; mobile interaction; audio interfaces.

\section{INTRODUCTION}

Walking has been shown in many studies to have an inverse correlation with body mass index (a measure of obesity) and to generally improve health [9][19][37]. Walking has also been shown to improve mental health and can alleviate symptoms of depression: for example, women who achieve over 7,500 steps per day have been shown to have a $50 \%$ lower prevalence of depression than women taking fewer than 5,000 [34]. Although precise targets vary, 7,000-8,000 steps per day is an appropriate health-enhancing walking guideline for adults [34] and many countries have now adopted steps/day as a target to help increase activity levels of their populations. However, in order to achieve full health benefits people must walk at a moderate intensity cadence, or pace, to achieve a sufficiently elevated metabolic rate. It is suggested that at least 3,000 of the steps per day be taken at a moderate to vigorous pace [34]. The target rate is often defined as 3 METs or higher (3 times the Metabolic Equivalent of a resting Task, roughly walking at $5 \mathrm{~km} / \mathrm{h}$ [15]). While varying per person, this "moderate activity" level is achieved on average by walking at or over $100 \mathrm{steps} / \mathrm{min}$ [35].
Our research targets individuals who wish to improve their health through walking but who currently fall short of the daily targets. Studies have shown that many people in this category have a normal walking pace below the required moderate pace and find it difficult to reach and maintain the required cadence: they tend to resort to their typical slower walking pace. Since many people who undertake exercise also like to listen to music at the same time, our aim is to investigate whether degradation of music on a mobile music player can act as intervention feedback to help people learn and maintain a target walking cadence. We have developed a novel approach based on real-time sound effects that degrade music quality and a prototype application that uses standard smartphone sensors to act as a pedometer and adjust sound quality dynamically. We use step-count information to reduce the perceived quality of walkers' own music when they drop below a target cadence, allowing users free choice of music to listen to (compared to previous work that targeted beats-perminute, as discussed below). Our aim is to achieve nonintrusive eyes-free ambient feedback that reminds people to walk at the right cadence and will help them learn that cadence.

\section{HEALTH IMPROVEMENT MOTIVATION}

In order for people to take part in any kind of physical exercise, it is necessary to motivate them and to provide feedback and encouragement to maintain their exercise. There has been considerable research into understanding and predicting our behaviour regarding health (e.g. [6]) and, in their work on persuasive game design, Orji et al. concluded that "Persuasive games that are informed by behavioural theories tend to be more successful than those based on intuition" [23]. Behaviour theory can help us understand motivation for and process of lifestyle change, e.g. through the Transtheoretical Model of Behaviour Change [25]. This approach models the stages that a person goes through in attempting a health behaviour change. Another approach is through understanding the role of self-belief or self-efficacy in supporting behaviour change (e.g. [30]). This approach focuses on self-esteem to achieve the behavioural change, on the premise that people with low confidence are less likely to take part in difficult activities like physical exercise. Finally, a further approach is through understanding the social aspects of health (e.g. [4]). Social factors can have a major impact on health and the perception that people can use the performance of others to motivate themselves, not only to start an activity but to keep it up over a period of time. For example, if more 
people in a population begin walking more then a higher daily step count may be regarded as the norm [24].

The Transtheoretical Model of Behaviour Change divides the fitness-activity lifecycle of a person into five stages: Precontemplation where the person does not even think (s)he needs a change in behaviour; Contemplation where the person realizes that he needs to change his behaviour; Preparation where the person prepares to act; Action where the person starts to change his behaviour and; Maintenance where the person keeps up the behaviour change. Our work here is focused on supporting the move from Preparation to Action for lower fitness level individuals.

\section{APPLICATIONS TO IMPROVE HEALTH}

Recently physical activity has become a crucial issue for game designers. There is a shift in the idea of gaming from a sedentary activity to a more active one where physical exercise becomes the central mechanic of the game [10][32]. Games to promote health can be categorized as: Educational games that can be used to learn about different diseases, nutrition and health; Activity Enhancing Games to enhance physical activity by making it more fun; and Persuasive games that attempt to persuade the users to modify their behaviour [1].

In the domestic setting the Nintendo Wii and Microsoft Kinect use body movements to simulate sports while arcade games that include physical movement are also popular, e.g. Dance-Dance-Revolution. As game-play is the focus for these applications, and not typically exercise, their main health benefits are probably in the precontemplation stage as users can play without having consciously considered lifestyle changes - the game can act as a 'gateway fitness' activity [29].

Pervasive games are games that are not confined to a board, device or room but take place outdoors. As an example: a pervasive treasure hunt would have clues distributed throughout an area which could be picked up by a mobile phone with user movement being an incidental requirement of playing the game. Gorgu et al. [11] coined exergaming to describe their game, luften, in which players achieve a number of objectives by moving around a university campus. Much exergaming activity has been based around this pervasive gaming model with users walking or running around the environment (e.g. [3]). Others have investigated dance [5] or mobile support for outdoor training equipment [2]. While not targeting a particular stage of the Transtheoretical Model, again these are likely to have biggest impact on precontemplation as the focus is more on the game than health.

More generally, there has been a plethora of applications and research into mobile applications to support health and pervasive gaming. In [14], a review of research into mobile phone applications for managing obesity from a user-centred design perspective examined over 50 applications targeting different aspects of mobile support for health and concluded on the need for research into peer-to-peer sharing, privacy and the use of behaviour change models Probably the most influential pervasive health research for the HCI community is that of Consolvo et al. [7]. Mainly targeting the central action and maintenance phases, the researchers identified four key design requirements for technologies that encourage physical activity: "(1) Give users proper credit for activities, (2) Provide personal awareness of activity level, (3) Support social influence, and (4) Consider the practical constraints of users' lifestyles." In the Fish ' $\mathrm{n}$ ' Steps [18] and UbiFit [8] projects, two innovative ambient displays were created to encourage healthier lifestyles: one based on fish growth in a shared tank and one on a garden growing as a mobile phone wallpaper. Both were primarily aimed at encouraging people to do exercise and to maintain that exercise level over time.

Building on the strong social links that encourage many joggers and runners to both become active and maintain their activities, Mueller et al. used audio links to give an impression of running together with a remote partner [20] while TripleBeat developed real-time competition with data from a collection of other runners [21].

\section{BEATCLEARWALKER AIMS}

In this research we are primarily targeting people in the early Action stage, in which walkers often do not know what the target cadence feels like and tend to walk too slowly for the main cardiovascular benefits of walking [33]. Many people wanting to exercise outdoors find that listening to music can help and encourage their walking [16]. Music has been shown to strongly affect exercise level of participants (e.g. [17]), for example in gym classes, participants can naturally increase their exercise level as the beat of the music increases. Early work on mobile health apps with music showed that these effects can be translated to runners wearing mobile phones [22]. Although there has been some success (e.g. [28][26]), for walking, the situation is less clear as it can be difficult to pick a varied and appropriate set of music of given rhythms from a walker's own music collection. Furthermore, a user's choice of music is likely to be largely affected by their mood, desires and personal taste, unlike music used for gym classes that is often deliberately selected for the exercising effect. It is also unclear whether the effect on users of changing beats-per-minute is as effective for walking as it is for more intensive exercise such as gym classes and running. As an alternative to rhythm we wanted to investigate the use of sound effects as feedback to users. Our aims were to:

- Provide rapid feedback to users to help them learn and maintain walking at an elevated cadence;

- Allow the users a free choice of music while walking;

- Allow eyes-free interaction with the device so that users can walk without looking at or holding their phone;

- Provide feedback that is noticeable but not excessively intrusive or disruptive to music listening;

- Support a wide range of music genres. 
While glanceable interfaces (e.g. [21]) and tactile feedback (e.g. [31]) have been used successfully in active lifestyle apps, in our work we decided to focus on the audio channel for eyes free interaction. We wanted to avoid spoken feedback and abstract additional sounds as these can interfere with music. Previous work has investigated the use of tempo changes and additional sounds on mobile devices to affect exercise (e.g. [12][22][27]). Our work differs from these, as we focus on changing the perceived quality of the sound as an ambient feedback that does not restrict the choice of music and targets walking cadence. We developed a model that allows users to listen to their own music while walking and gives audio feedback as follows:

- Clear - while the user is walking at or above their target cadence their music will be played normally as if listening to a normal MP3 player on their mobile phone;

- Degraded - when the walker's cadence drops below the threshold we aim to degrade the music quality with a perceptible, but not overwhelming, audio effects applied to the music. These effects will clear once the user's cadence reaches target level.

This paper presents our investigation into the research question: Can music degradation be used to help users learn and maintain an elevated cadence? We developed an Android smartphone application that monitored cadence and dynamically applied degradation effects to music. We ran initial trials of the system to gain insights into the quality of step detection and the effectiveness of various audio effects over a range of music. Finally, we ran controlled user trials with 20 participants walking in an outdoor setting, to assess if the application can help maintain a target elevated cadence.

\section{INITIAL STUDIES}

In order to build an accurate pedometer and a music player capable of providing effective audio cues, we initially developed two prototypes for evaluation. The results from this testing helped shape the end application by investigating the quality of the accelerometer-based pedometer and the noticeability of different music affects.

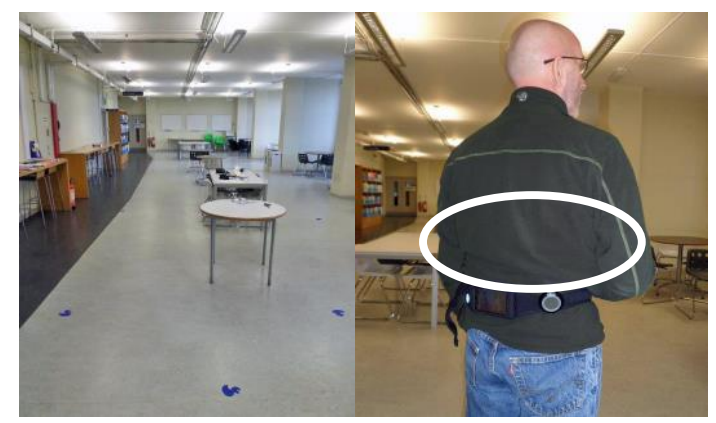

Figure 1: Study space and user with devices

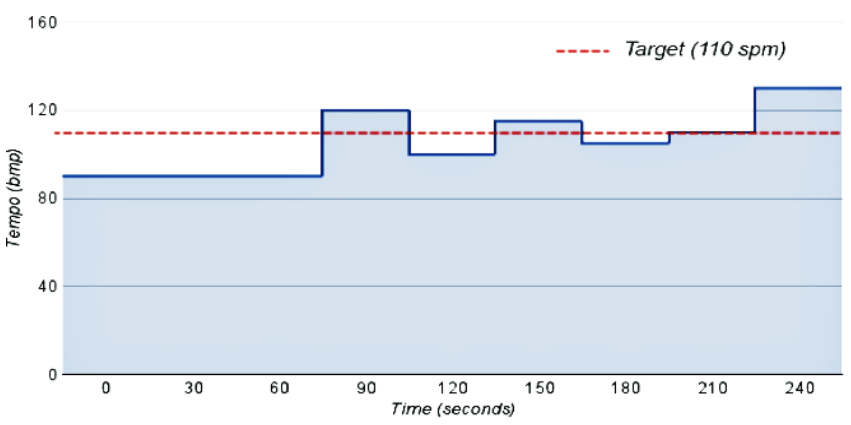

Figure 2: Tempo pattern of test track

To select a high quality detection algorithm for our accelerometer based step counter we compared the number of steps detected through hand-counting video data (the 'gold standard') with the analysis of the same walks using several peak detection algorithms in Matlab. The algorithms were compared and PeakFinder ${ }^{1}$ had the best performance. We adapted this algorithm to work in real-time on the device using a 5-second window of samples from the built-in accelerometers. This prototype was tested with seven users using their own Android devices. Each participant was asked to walk at three different cadences around a normal target rate (90, 110, and 130 steps per minute) for two minutes each around a circuit in an indoor university study area (see Figure 1 left). The pedometer prototype was first installed on their phones and placed in the user's pocket. A second phone was strapped onto their back along with a commercial pedometer (New Lifestyles NL-1000) (Figure 1 right). For baseline we also manually counted steps walked during the 2-minute study periods. Users were asked to walk in time to the beat from an electronic metronome. This study showed that we were able to measure with similar accuracy to the commercial pedometer (around $+/-6 \%$ variation from manual counting, where a variation of $+/-10 \%$ is acceptable for an "accurate pedometer" [36]). However, we identified a problem with variations in behaviour between devices and decided to limit further studies to a single device. To confirm our findings, we also ran tests by asking skilled walking researchers to walk in time to the beat from an electronic metronome following a pre-defined beatpattern (see Figure 2) that tested a range of walking paces around the normal walking target of $110 \mathrm{step} / \mathrm{min}$. This confirmed our application as achieving the $+/-10 \%$ threshold compared to manual counting by trained observer.

We investigated a range of options available to affect music quality in real time. To minimize load on the phone, we investigated use of the inbuilt Android Media AudioFx SDK that allows dynamically adjusting equalizer and reverb levels, along with applying a "bass boost". A prototype interface was created, allowing a range of equalizer and reverb settings to be changed at runtime. The Reverb effects made little difference to the output; however there were noticeable changes to the

\footnotetext{
${ }^{1}$ www.mathworks.co.uk/help/dsp/ref/peakfinder.html
} 
output by lowering the decibel level of the five frequency bands available. We investigated three presets that lowered different bands: Effect 1 removed the lowest frequency bands, making it difficult to hear the bass beat from a track. Effect 2 removed the lowest and highest bands, so high keyboard notes and vocals were also harder to hear. Effect 3 removed a mixture of high, mid, and low frequencies, leaving only the main bass line and a little melody. Seven users were asked to listen to three different genres of music through the application while we applied the different effects. Effects 1 and 2 were not noticeable for country and some metal music, whereas effect 3 was noticeable for all genres, so it was decided to utilize this effect in the final implementation. The effect was, however, still relatively subtle, particularly when using basic mobile earphones. As such we decided to augment effect 3 with pink noise to emulate a badly tuned AM radio.

\section{BeatClear Walker Prototype}

Based on the initial studies our prototype BeatClearWalker application (Figure 4) monitors steps using PeakFinder. For experimental control it allows the target cadence to be set, a metronome to play initially to help establish the target cadence and audio effects based on adjusting equalizer settings and introducing a volume-controllable amount of pink noise (set at $20 \%$ of music volume in studies).

\section{EVALUATION STUDIES}

A formal evaluation was carried out to gauge the impact of the BeatClearWalker in supporting people walking at an pace above their typical walking pace and to gain insights into users' impressions of using the application. Our study had two hypotheses:

- H1: Users will better maintain their target cadence with our degradable player than a plain player;

- H2: Effects of learning the target cadence will have some persistence when effects are removed.

\section{A. Experimental Design}

Participants were recruited in Patras using personal contacts and email lists. We recruited 20 participants (11 male, 8 female; 18 were in the $18-30$ age group, and two were in the 50-59 group). To encourage participation, the participants were entered into a random draw to win a Nexus 7 tablet. Studies were conducted under University of Strathclyde ethical approval. In particular we highlighted that participants should abandon the study at any point if they felt pain or any discomfort. Users initially completed a simple background form and then conducted four walks in a pedestrian only harbour-side location of Patras (see Figure 3). Our app ran on quad-core Android phones, placed in the participant's trouser pockets or mounted on a waist belt (in cases where pockets were not present). The stages were as follows:

1. Judging walker's typical pace: To set the target elevated pace we needed to first assess each walker's typical

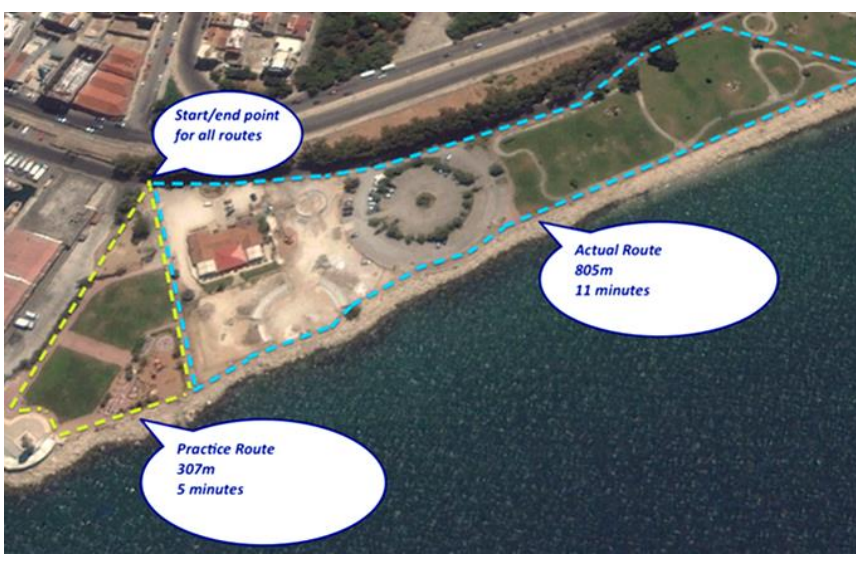

Figure 3: Study routes in [ blinded] with distances and approximate times

walking pace. We first asked users to walk a route without music at their normal pace (in line with [22] and standard walking study practice [35]). Each participant was asked to walk a triangular route from the starting point, keeping a steady and comfortable pace. The approximate route length was $307 \mathrm{~m}$, which we estimated would take around 5 minutes to walk. We recorded steps using the phone and the time taken to complete the lap using a stopwatch. We converted steps taken into METs using the participants' self-reported height and a standard table of cadence-MET conversion.

2. Learning the target pace: Our study aim was to assess walkers' ability to walk at an elevated pace with and without our music effects. We set this elevated pace as follows: if the user's initial walk was performed at a pace under 4.0 METs then the target for the subsequent study phases (phases 2 and 3) was set at their pace plus 1 MET an achievable faster than normal walking pace. As it can

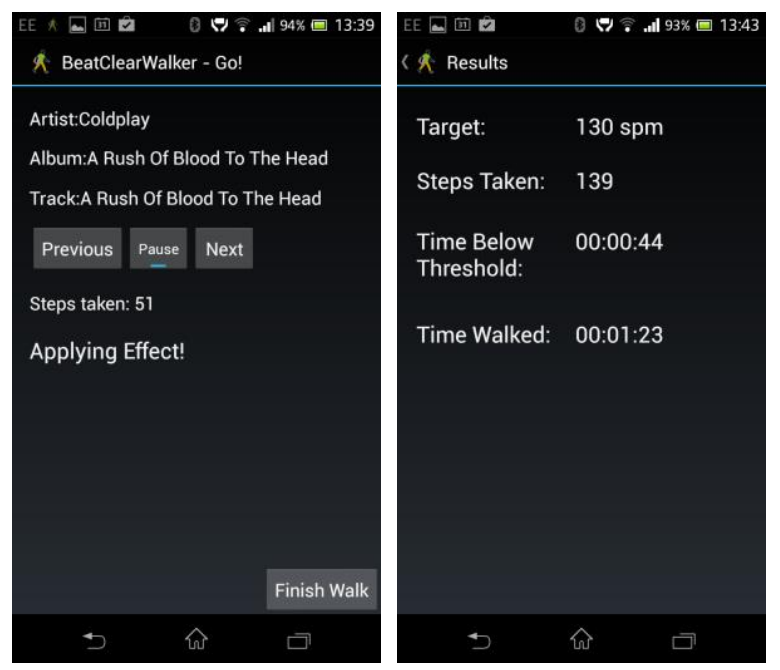

Figure 4: BeatClearWalker (left: while walking, right: report screen) 
be physically difficult to achieve over 5 METs by walking we decided to cap the target pace at 5 METs for any participant who performed the initial walk at $>=4$ METs (in our study, none did). For this walk BeatClearWalker was set up with a metronome beating at a tempo equal to the user's target cadence, but without music. The participant was asked to repeat the practice route at a cadence that matched the tempo of the metronome and asked to focus on that cadence and remember it for subsequent walks.

3. Music walk 1 and 2: We investigated two study conditions in a balanced within-subject design. The two conditions were a plain music player $(\mathrm{P})$ and a degradable music player (D), both running on the same phone using BeatClearWalker and a mixed BPM adult contemporary playlist. The conditions were balanced with half the users starting on P (Group PD) and half on D (Group DP). For each condition the user was asked to walk a $0.8 \mathrm{~km}$ path (estimated at about 11 minutes walking time) at the established pace from walk 2 while listening to music. For condition D we informed participants that the music would degrade if they dropped below the target pace, and that in order to maintain the target cadence, they would need to increase their walking cadence, at which point the full quality music would return. In condition $\mathrm{P}$, music was played without these effects. In both cases the metronome played briefly at the start to remind the walker of the desired pace and then stopped after 16s. We measured their speed per lap and cadence per minute for these tests steps using the phone software, lap time by stopwatch.

After each main walk users completed a post-study questionnaire to assess workload and at the end of the study to assess their impression of the two systems. Finally, in line with standard wellbeing study practice, users were given an information and feedback sheet on walking cadence, health benefits, and a simple interpretation of their "typical" cadence in relation to this information.

\section{B. Quantitative Experimental Results - Overall}

For each participant the mobile app captured the total number of steps taken, the duration of the walk and the time which the participant walked below their target pace. Postexperiment, we calculated for each participant the steps walked under the desired pace as a percentage of the walk duration. The analysis that follows concerns the results of phase 3 (walks 3 and 4) as the first two walks were used only to establish the experimental conditions. Steps taken and walk times were approximately normally distributed (Shapiro-Wilk) while time walked (count and fraction) under target rate differed significantly from normal distribution. As such repeat measure ANOVA was used for analysis of steps taken and total time, while Wilcoxon Signed Ranks was used for time under target.
Our study design asked participants to complete a fixed distance in two conditions. Figure 5 shows that overall users took slightly, but significantly, longer to complete the distance

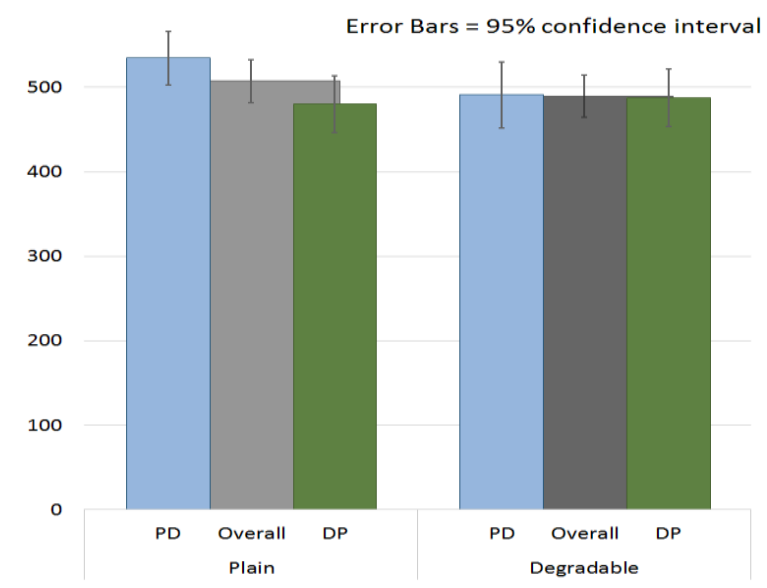

Figure 5: Total time for study (phase 3) walks

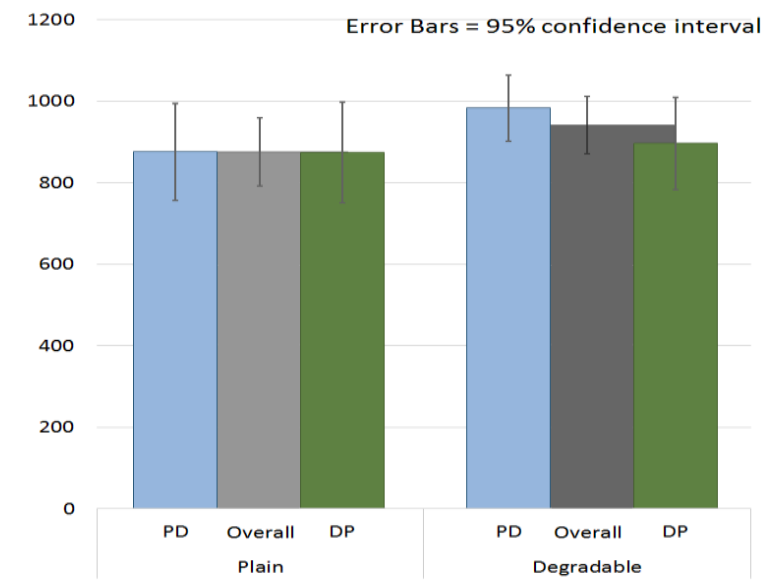

Figure 6: Number of steps taken during the walks

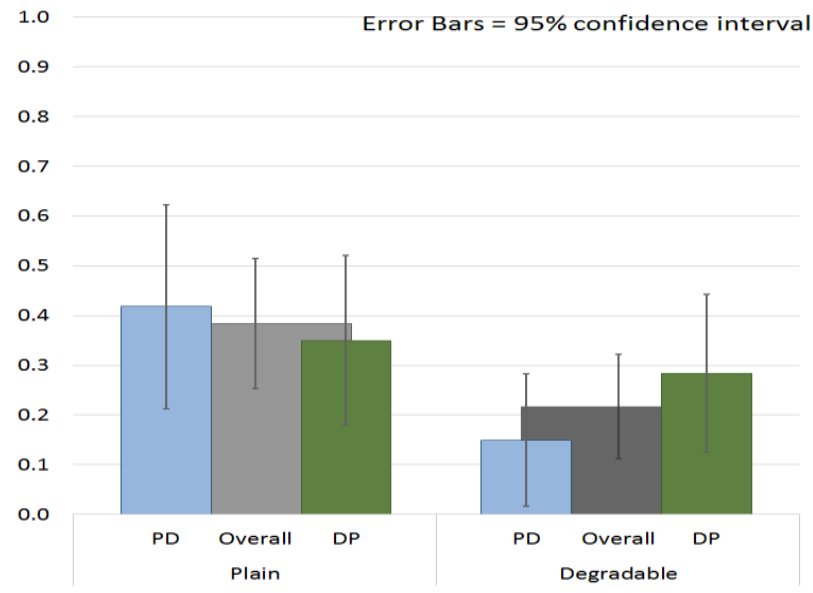

Figure 7: Fraction of steps below threshold 
with the plain music player (P) than the degradable player (D) $\left(F_{1,19}=4.515, \mathrm{p}=.047 ; \mathrm{M}_{\mathrm{P}}=507 \mathrm{~s} \mathrm{SD}_{\mathrm{P}}=59, \mathrm{M}_{\mathrm{D}}=490 \mathrm{~s} \mathrm{SD}_{\mathrm{D}}=57\right)$.

Our subjects also took significantly more steps (Figure 6) with our degradable music player than with the plain music player $\left(F_{1,19}=7.625, \quad \mathrm{p}=.012 ; \quad \mathrm{M}_{\mathrm{P}}=876 \quad \mathrm{SD}_{\mathrm{P}}=190, \mathrm{M}_{\mathrm{D}}=941\right.$ $\left.\mathrm{SD}_{\mathrm{D}}=161\right)$.

Finally we examined the time participants spent below their desired cadence threshold. We observed a statistically significant difference on the total time below each user's desired pace, with users dropping below the desired pace for longer in condition $\mathrm{P}$ than $\mathrm{D}\left(\mathrm{F}_{1,19}=11.911, \mathrm{p}=0.003 ; \mathrm{M}_{\mathrm{P}}=196\right.$ $\mathrm{SD}_{\mathrm{P}}=157, \mathrm{M}_{\mathrm{D}}=107, \mathrm{SD}_{\mathrm{D}}=120$ ). This is echoed in the fraction of time users walked below their threshold $(\mathrm{Z}=-3.40 \mathrm{p}=.001$; $\left.\mathrm{M}_{\mathrm{P}}=0.384 \mathrm{SD}_{\mathrm{P}}=0.298, \mathrm{M}_{\mathrm{D}}=0.217, \mathrm{SD}_{\mathrm{D}}=0.240\right)$.

In summary: overall users took more steps and achieved the target goal cadence for longer using the degradable music player (D) than a plain music player (P). This confirms our primary hypothesis $\mathrm{H} 1$ that music degradation can be used to help users maintain an elevated cadence.

\section{Quantitative Experimental Results - Learning Effects}

As well as the overall comparison between the two conditions, we were also interested in learning effects - in particular can the benefits from exposure to the degradable music player be sustained after the effects are removed? While a true longitudinal study is beyond the scope of this paper, looking at Figures 5-7 it is clear that Groups PD and DP differ, indicating a potential asymmetric learning effect.

Figure 6 shows that group PD (plain followed by degrading player) walked considerably, and significantly, more steps in condition $\mathrm{D}$ than $\mathrm{P} \quad\left(\mathrm{F}_{1,9}=6.15 \mathrm{p}=.035 ; \mathrm{M}_{\mathrm{P}}=877 \quad \mathrm{SD}_{\mathrm{P}}=191\right.$, $\left.\mathrm{M}_{\mathrm{D}}=984 \mathrm{SD}_{\mathrm{D}}=131\right)$. For Group $\mathrm{DP}$, condition $\mathrm{D}$ again resulted in significantly more steps being taken but with a marginal difference $\left(F_{1,9}=8.91 \mathrm{p}=.015 ; \mathrm{M}_{\mathrm{P}}=876 \mathrm{SD}_{\mathrm{P}}=199, \mathrm{M}_{\mathrm{D}}=898\right.$, $\mathrm{SD}_{\mathrm{D}}=183$ ).

Walkers in group PD took significantly longer on the walk with the plain player than the degradable player (Figure 5) $\left(F_{1,9}=26.9, p=.001 ; M_{P}=535 \mathrm{~s} \mathrm{SD}_{\mathrm{P}}=51, \mathrm{M}_{\mathrm{D}}=491 \mathrm{~s} \quad \mathrm{SD}_{\mathrm{D}}=63\right)$. This, along with the increased number of steps, shows that our Group PD participants walked faster under condition D. There was no significant difference in walking time for Group DP $\left(F_{1,9}=0.067, \mathrm{p}=.434 ; \mathrm{M}_{\mathrm{P}}=480 \mathrm{~s} \mathrm{SD}_{\mathrm{P}}=54, \mathrm{M}_{\mathrm{D}}=488 \mathrm{~s}, \mathrm{SD}_{\mathrm{D}}=55\right)$. We note, however, that these times are very close to those of condition D for Group PD.

We also examined the time participants walked below their desired pace threshold. Group PD walkers spent significantly and considerably longer under the threshold cadence in condition $\mathrm{P}$ than $\mathrm{D}\left(\mathrm{Z}=-2.70 \mathrm{p}=.007 ; \mathrm{M}_{\mathrm{P}}=224 \mathrm{~s} \quad \mathrm{SD}_{\mathrm{P}}=178\right.$, $\mathrm{M}_{\mathrm{D}}=77 \mathrm{~s}, \mathrm{SD}_{\mathrm{D}}=117$ ). In Group $\mathrm{DP}$, there was no significant difference between players $\left(Z=-1.68 \quad \mathrm{p}=.092 ; \quad M_{P}=169 \mathrm{~s}\right.$ $\left.\mathrm{SD}_{\mathrm{P}}=136, \mathrm{M}_{\mathrm{D}}=136 \mathrm{~s}, \mathrm{SD}_{\mathrm{D}}=121\right)$. A similar pattern was observed for the fraction of steps under the threshold: Group PD showed significantly lower fraction under threshold with player D than $\mathrm{P}$, see Figure $7\left(\mathrm{Z}=-2.55 \mathrm{p}=.011 ; \mathrm{M}_{\mathrm{P}}=0.419\right.$ $\mathrm{SD}_{\mathrm{P}}=0.337, \mathrm{M}_{\mathrm{D}}=0.148, \mathrm{SD}_{\mathrm{D}}=0.214$ ); a much smaller, albeit significant, difference was observed for group DP walkers $(\mathrm{Z}=-$ $\left.2.15 \mathrm{p}=.032 ; \mathrm{M}_{\mathrm{P}}=0.350 \mathrm{SD}_{\mathrm{P}}=0.275, \mathrm{M}_{\mathrm{D}}=0.285, \mathrm{SD}_{\mathrm{D}}=0.257\right)$.

In summary: both groups took less time in condition $\mathrm{D}$ but the effect sizes were smaller for those exposed to the degradable player first. Those first exposed to the degradable player walked less below the target threshold and walked more steps in total, but there was marginal difference for those using plain after degradable. As such, we conclude that the training effect of exposure to the degradable player carried on into the plain player condition giving evidence that users had learned the target pace during the degrading test and could carry this on afterwards - supporting $\mathrm{H} 2$ over a short-term.

\section{Workload and Qualitative Experimental Results}

After each study walk (3 and 4) participants completed a NASA-TLX questionnaire [13] to capture their subjective experience of the walk (Figure 9). For statistical significance tests we used Wilcoxon signed rank tests throughout for consistency as four of the categories differed significantly from normal in one condition (Shapiro-Wilk test).

Walkers reported a relatively low mental effort for both conditions, with a slightly higher, but non-significant, effort for the plain player $\left(Z=-1.83 \mathrm{p}=.068 \mathrm{~N}=20 ; \mathrm{M}_{\mathrm{P}}=5.15 \mathrm{SD}_{\mathrm{P}}=2.03\right.$, $\left.\mathrm{M}_{\mathrm{D}}=4.10 \mathrm{SD}_{\mathrm{D}}=1.59\right)$. With regard to the physical effort, overall participants reported a significantly higher physical effort while using the degradable player $(Z=-3.761 \mathrm{p}=.000 \mathrm{~N}=20$; $\left.\mathrm{M}_{\mathrm{P}}=13.00 \mathrm{SD}_{\mathrm{P}}=1.97, \mathrm{M}_{\mathrm{D}}=15.6 \mathrm{SD}_{\mathrm{D}}=1.28\right)$. As the dominant physical activity was walking, we conclude they felt that they were walking harder with the degradable player. Participants also felt under significantly greater temporal demand with the degradable player, implying a less relaxed activity $(\mathrm{Z}=-3.672$ $\mathrm{p}=.000 \mathrm{~N}=20 ; \mathrm{M}_{\mathrm{P}}=11.90 \mathrm{SD}_{\mathrm{P}}=2.25, \mathrm{M}_{\mathrm{D}}=15.25 \mathrm{SD}_{\mathrm{D}}=1.92$ )

There was no significant difference in the users' ratings of their level of performance between the two conditions ( $\mathrm{Z}=-$ $1.29 \mathrm{p}=.196 \mathrm{~N}=20 ; \quad \mathrm{M}_{\mathrm{P}}=13.85 \quad \mathrm{SD}_{\mathrm{P}}=2.75, \quad \mathrm{M}_{\mathrm{D}}=14.95$ $\mathrm{SD}_{\mathrm{D}}=2.09$ ). There was a small difference (indicative but not significant) in reported overall effort with the degradable player being slightly higher overall $(\mathrm{Z}=-1.885 \mathrm{p}=.059 \mathrm{~N}=20$; $\left.\mathrm{M}_{\mathrm{P}}=13.65 \mathrm{SD}_{\mathrm{P}}=2.68, \mathrm{M}_{\mathrm{D}}=15.15 \mathrm{SD}_{\mathrm{D}}=1.95\right)$.

Finally on workload assessment, frustration was below average for both conditions, indicating that our application was well received and enjoyable $\left(\mathrm{Z}=-0.430 \mathrm{p}=.667 \mathrm{~N}=20 ; \mathrm{M}_{\mathrm{P}}=7.15\right.$ $\mathrm{SD}_{\mathrm{P}}=1.46, \mathrm{M}_{\mathrm{D}}=7.50 \mathrm{SD}_{\mathrm{D}}=2.42$ ).

In summary users did not feel they were working hard mentally but that they were working quite hard physically and under time pressure. Furthermore, they reported working harder physically and under more time pressure when using the degradable player. They did not perceive a significant difference in performance (which they rated as fairly high) nor in frustration (which they rated fairly low). 
At the end of the study we asked participants six general feedback questions, in order to capture their overall experience and attitude towards our degrading player. The questions were on a 7-point Likert scale as follows:

Q1. Did you find it easier to walk at the right pace with or without sound effects? (much easier 1 to 7 much easier without)

Q2. Did you feel the sound effects helped you learn the faster pace we asked you to walk at? (1 considerably, 7 not at all)

Q3. For casual walks (e.g. to the shops) do you see yourself using these sound effects to increase your walking pace and exercise level? (1 regularly, 7 never)

Q4. For exercise walks ... do you see yourself using these sound effects to increase your walking pace and exercise level? (1 regularly, 7 never)

Q5. If your normal MP3 player could do these sound effects while you are walking, would you use it? (1 always, 7 never)

Q6. If a dedicated app was available, would you recommend this to friends / family? (1 highly, 7 not at all).

From the users' responses (Figure 8), we note that users found it easier overall to walk at the right pace with our degrading player $(\mathrm{M}=2.15, \mathrm{SD}=0,75)$ and also felt that the sound effects helped them in learning the required pace $(\mathrm{M}=1.90, \mathrm{SD}=0.72)$. Participants also felt that our system could be used sometimes for casual walks $(\mathrm{M}=3.1, \mathrm{SD}=0.85)$ but was probably better suited for dedicated exercise walks $(M=1.6$, $\mathrm{SD}=0.75$ ). This is corroborated by their response to having such effects incorporated in their normal MP3 player, where participants were inclined towards not using them $(M=4.15$, $\mathrm{SD}=0.81$ ), showing that perhaps a separate app would be more desirable than incorporating degradation as a feature in an existing player but if such an app existed, participants would highly recommend it to family and friends $(\mathrm{M}=1.3, \mathrm{SD}=0.47)$.

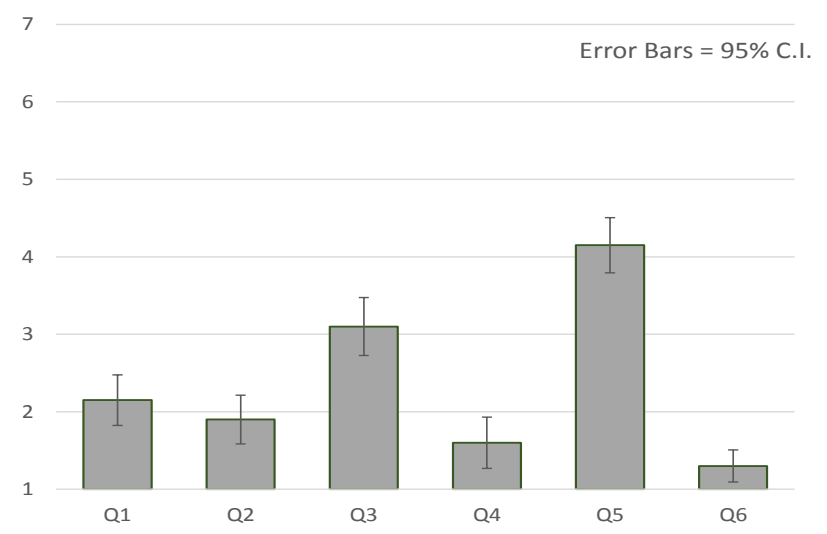

Figure 8: Final feedback questionnaire responses

Users also give more general feedback in response to an open final question on the exercise being effective (e.g. "This is more exercise than I thought I would get for such a short walk.", "I didn't expect to actually begin to sweat!") their appreciation of the feedback mechanism (e.g. "I struggled to remember the pace without the feedback", "the feedback is good, I prefer it over the nagging reminders of a computerized voice", "I think this app would have really helped us when we started [walking for exercise a couple of months ago] because we would usually get distracted chatting", "degradation of music is less annoying [than metronome]") and some limitations of the system (e.g. "it might be difficult to perceive the feedback in a noisy environment", "the app should understand when you've come to a traffic light and are waiting.") [all quotes translated to English].

\section{STUDY LIMITATIONS}

We believe that this paper presents the first results showing that non-intrusive audio feedback can affect adherence to target walking cadence, however the study only looked at one form of feedback and it would be useful to research aspects of our feedback in more detail (e.g. looking at variations of sound effects and at other types of audio and/or tactile feedback).

The study was relatively short, particularly for assessing learning effects, and although conducted outdoors the environment was fairly stable. Users also listened to our music rather their own for consistency. Further addressing of both of these shortcomings is part of our future work.

\section{CONCLUSIONS AND FUTURE WORK}

Our research has shown that users can detect music that has been degraded using equalizer and noise effects. We have identified particular effects that perform well across a range of music genres. Using a real-time variant of PeakFinder we have developed a pedometer for Android smartphones that performs to a similar standard as commercial pedometers. We combined these two systems into BeatClearWalker - a mobile application that degrades perceived music quality when walking below a target cadence. We conducted studies of BeatClearWalker with 20 participants walking for a total of approximately $2.2 \mathrm{~km}$ each. These studies showed that our music effects reduced the time walked below a target elevated walking cadence. There was also evidence of an asymmetric learning effect from using BeatClearWalker, in that subsequent to using the degrading function people appeared to be able to maintain a target walking pace when the music degradation function was used not enabled. Workload assessment showed that users were not working hard mentally but were working hard physically and felt they worked harder and were under more time pressure when using the degradable music player.

These results support our research questions that music degradation be used to help users maintain and learn an elevated cadence. In post-study questionnaires users were positive about the effects and said they would use them for exercise walks (and to a lesser extent for casual walks).

We are currently further developing the application for release - in particular we are further tuning the accuracy of the pedometer, fully integrating it with users' own music libraries and improving performance across different devices. We will 
then use this deployed application for longitudinal studies to understand how it is used in practice, for whom it is most effective and the long term effects of the player. One particular aspect of the current application is that it does not detect stopping, for example to wait to cross the road. While this is useful for feedback that the system is working and monitoring it may be annoying long term despite the relatively subtle effects and may also prevent usage as a person's normal player. We are also investigating how to calibrate the effects to an individual's own phone and headset. In related research we are also studying how people want to record and review their success rates and potential linkage to social networking sites. We are also interested in how BeatClearWalker can be used to give feedback on target adherence in other exercise activities.

More widely, directly manipulating sound quality has been researched surprising little. Our work has shown that it can be used for feedback while people are listening to music. Although relatively low bandwidth feedback, we believe that use of sound effects could be used more widely in interface design to give non-intrusive eyes-free feedback to users on real time performance. Our users clearly noticed and responded to the feedback and did not find it excessively annoying.

\section{ACKNOWLEDGEMENTS}

We are grateful for the time and effort of our participants in the early studies and main walking study. We are also grateful to colleagues in both departments in Strathclyde and at The University of Patras for supporting the project.

\section{REFERENCES}

1. Brox, E., Fernandez-Luque, L., and Tøllefsen, T. Healthy Gaming-Video Game Design to promote Health. Appl Clin Inf 2, (2011).

2. Buttussi, F., Chittaro, L., and Nadalutti, D. Bringing Mobile Guides and Fitness Activities Together: A Solution Based on an Embodied Virtual Trainer. Proc MobileHCI 2006, ACM (2006).

3. Cheok, A.D., Goh, K.H., Liu, W., et al. Human Pacman: a mobile, widearea entertainment system based on physical, social, and ubiquitous computing. Personal \& Ubiquitous Comp. 8, 2 (2004).

4. Christakis, N.A. and Fowler, J.H. The spread of obesity in a large social network over 32 years. New England journal of medicine 357, 4 (2007).

5. Clawson, J., Patel, N., and Starner, T. Dancing in the Streets: The design and evaluation of a wearable health game. Proc ISWC, (2010).

6. Conner, M. and Norman, P. Predicting health behaviour. McGraw-Hill International, 2005.

7. Consolvo, S., Everitt, K., Smith, I., and Landay, J.A. Design Requirements for Technologies That Encourage Physical Activity. Proc. SIGCHI, ACM (2006).

8. Consolvo, S., McDonald, D.W., Toscos, T., et al. Activity Sensing in the Wild: A Field Trial of Ubifit Garden. Proc. SIGCHI, ACM (2008).

9. Craig, R. and Hirani, V., eds. Health Survey for England - 2009: Health and lifestyles. The NHS Information Centre, 2010.

10. Ferguson, B. The emergence of games for health. GAMES FOR HEALTH: Res., Dev., and Clinical Applications 1, 1 (2012).

11. Gorgu, L., O'Hare, G.M.P., and O'Grady, M.J. Towards Mobile Collaborative Exergaming. Advances in Human-oriented and Personalized Mechanisms, Tech., and Services. Proc. CENTRIC (2009).

12. Hartnett, J., Lin, P., Ortiz, L., and Tabas, L. A Responsive and Persuasive Audio Device to Stimulate Exercise and Fitness in Children. Proc. SIGCHI, ACM (2006).
13. Hart, S.G. and Staveland, L.E. Development of NASA-TLX (Task Load Index): Results of Empirical and Theoretical Research. In Peter A. Hancock and Najmedin Meshkati, ed., Adv. in Psychology. 1988.

14. Hermawati, S. and Lawson, G. Managing obesity through mobile phone applications: a state-of-the-art review from a user-centred design perspective. Personal and Ubiquitous Computing, (2014).

15. Jetté, M., Sidney, K., and Blümchen, G. Metabolic equivalents (METS) in exercise testing, exercise prescription, and evaluation of functional capacity. Clinical Cardiology 13, 8 (1990), 555-565.

16. Karageorghis, C.I., Mouzourides, D.A., Priest, D.-L., Sasso, T.A., Morrish, D.J., and Walley, C.L. Psychophysical and ergogenic effects of synchronous music during treadmill walking. J. Sport \& Exercise Psychology 31, 1 (2009).

17. Karageorghis, C.I. and Terry, P.C. The psychological, psychophysical and ergogenic effects of music in sport: A review and synthesis. (2008).

18. Lin, J.J., Mamykina, L., Lindtner, S., Delajoux, G., and Strub, H.B. Fish'N'Steps: Encouraging Physical Activity with an Interactive Computer Game. Proc. UbiComp, Springer-Verlag (2006).

19. Manson, J.E., Hu, F.B., Rich-Edwards, J.W., et al. A prospective study of walking as compared with vigorous exercise in the prevention of coronary heart disease in women. New England J. Medic. 341, 9 (1999).

20. Mueller, F. "Floyd," O'Brien, S., and Thorogood, A. Jogging over a Distance: Supporting a "Jogging Together" Experience Although Being Apart. Proc. SIGCHI, ACM (2007).

21. De Oliveira, R. and Oliver, N. TripleBeat: Enhancing Exercise Performance with Persuasion. Proc MobileHCI 2006, ACM (2008).

22. Oliver, N. and Flores-Mangas, F. MPTrain: a mobile, music and physiology-based personal trainer. Proc MobileHCI, ACM (2006).

23. Orji, R., Mandryk, R.L., Vassileva, J., and Gerling, K.M. Tailoring Persuasive Health Games to Gamer Type. Proc. SIGCHI, ACM (2013).

24. Pooley, C., Tight, M., Jones, T., et al. Understanding walking and cycling: Summary of key findings and recommendations. (2011).

25. Prochaska, J.O. and DiClemente, C.C. Transtheoretical therapy: Toward a more integrative model of change. Psychotherapy: Theory, Research \& Practice 19, 3 (1982).

26. Qian, H., Kuber, R., Sears, A., and Murphy, E. Maintaining and modifying pace through tactile and multimodal feedback. Interacting with Computers 23, 3 (2011).

27. Rowe, D., McMinn, D., Peacock, L., et al. Cadence, Energy Expenditure and Gait Symmetry During Music-Prompted and Self-Regulated Walking in Adults with Unilateral Transtibial Amputation. Journal of physical activity \& health, (2013).

28. Schauer, M. and Mauritz, K.-H. Musical motor feedback (MMF) in walking hemiparetic stroke patients: randomized trials of gait improvement. Clinical Rehabilitation 17, 7 (2003).

29. Schwanda, V., Ibara, S., Reynolds, L., and Cosley, D. Side Effects and "Gateway" Tools: Advocating a Broader Look at Evaluating Persuasive Systems. Proc. SIGCHI, ACM (2011).

30. Schwarzer, R. Self-efficacy in the adoption and maintenance of health behaviors: Theoretical approaches and a new model. Hemisphere Publishing Corp, 1992.

31. Spelmezan, D. An Investigation into the Use of Tactile Instructions in Snowboarding. Proc MobileHCI 2006, ACM (2012).

32. Stach, T.B. Design aspects of multiplayer exergames. Queen's University, Canada, 2010.

33. Tudor-Locke, C., Camhi, S.M., Leonardi, C., et al. Patterns of adult stepping cadence. Preventive medicine 53, 3 (2011).

34. Tudor-Locke, C., Craig, C.L., Brown, W.J., et al. How many steps/day are enough?. Int J Behav Nutr Phys Act 8, 1 (2011).

35. Tudor-Locke, C. and Rowe, D.A. Using cadence to study free-living ambulatory behaviour. Sports Medicine 42, 5 (2012).

36. Vincent, S.D. and Sidman, C.L. Determining measurement error in digital pedometers. Measurement in Phys. Edu. \& Exercise Science 7, 1 (2003).

37. Wagner, A., Simon, C., Ducimetiere, P., et al. Leisure-time physical activity and regular walking or cycling to work are associated with adiposity and 5 y weight gain in middle-aged men: the PRIME Study. Int. J. Obesity \& Related Metabolic Disorders 25, 7 (2001). 